\title{
Value of Retrospective Fusion of PET and MR Images in Detection of Hepatic Metastases: Comparison with ${ }^{18}$ F-FDG PET/CT and Gd-EOB-DTPA-Enhanced MRI
}

Olivio F. Donati ${ }^{1}$, Thomas F. Hany ${ }^{2}$, Caecilia S. Reiner ${ }^{1}$, Gustav K. von Schulthess ${ }^{2}$, Borut Marincek ${ }^{1}$, Burkhardt Seifert ${ }^{3}$, and Dominik Weishaupt ${ }^{1}$

${ }^{1}$ Institute of Diagnostic Radiology, University Hospital Zurich, Zurich, Switzerland; ${ }^{2}$ Division of Nuclear Medicine, University Hospital Zurich, Zurich, Switzerland; and ${ }^{3}$ Biostatistics Unit, Institute of Social and Preventive Medicine, University of Zurich, Zurich, Switzerland

The purpose of this study was to compare the accuracy of lesion detection and diagnostic confidence between ${ }^{18} \mathrm{~F}-\mathrm{FDG}$ PET/CT, gadolinium-ethoxybenzyl-diethylenetriamine pentaacetic acid (Gd-EOB-DTPA)-enhanced MRI, and retrospectively fused PET and MRI (PET/MRI). Methods: Thirty-seven patients (mean age $\pm \mathrm{SD}, 60.2 \pm 12 \mathrm{y}$ ) with suspected liver metastases underwent PET/CT and Gd-EOB-DTPA-enhanced MRI within 0-30 d (mean, $11.9 \pm 9$ d). PET and Gd-EOB-DTPA-enhanced MR image data were retrospectively fused. Images were reviewed independently by 2 readers who identified and characterized liver lesions using PET/CT, Gd-EOB-DTPA-enhanced MRI, and PET/MRI. Each liver lesion was graded on a 5-point confidence scale ranging from definitely benign (grade of 1) to definitely malignant (grade of 5). The accuracy of each technique was determined by receiver-operating-characteristic analysis. Histopathology served as the standard of reference for all patients with malignant lesions. Results: A total of 85 liver lesions (55 liver metastases [65\%] and 30 benign lesions [35\%]) were present in $29(78 \%)$ of the 37 patients. Twenty-four $(65 \%)$ of the 37 patients had liver metastases. The detection rate of liver lesions was significantly lower for PET/CT than for Gd-EOBDTPA-enhanced MRI (64\% and $85 \% ; P=0.002)$. Sensitivity in the detection and characterization of liver metastases for PET/CT, Gd-EOB-DTPA-enhanced MRI, PET/MRI in reader 1, and $\mathrm{PET} / \mathrm{MRI}$ in reader 2 was $76 \%, 91 \%, 93 \%$, and $93 \%$, respectively; the respective specificity values were $90 \%, 100 \%, 87 \%$, and $97 \%$. The difference in sensitivity between PET/CT and $\mathrm{PET} / \mathrm{MRI}$ was significant $(P=0.023)$. The level of confidence regarding liver lesions larger than $1 \mathrm{~cm}$ in diameter was significantly higher in PET/MRI than in PET/CT $(P=0.046)$. Accuracy values (area under the receiver-operating-characteristic curve) for PET/ CT, Gd-EOB-DTPA-enhanced MRI, PET/MRI in reader 1, and $\mathrm{PET} / \mathrm{MRI}$ in reader 2 were $0.85,0.94,0.92$, and 0.96 , respectively. Conclusion: The sensitivity of Gd-EOB-DTPA-enhanced $\mathrm{MRI}$ and $\mathrm{PET} / \mathrm{MRI}$ in the detection of liver metastases is higher than that of PET/CT. Diagnostic confidence was significantly bet-

Received Jul. 17, 2009; revision accepted Jan. 15, 2010.

For correspondence or reprints contact: Olivio F. Donati, Institute of Diagnostic Radiology, University Hospital Zürich, Rämistrasse 100, $\mathrm{CH}-8091$ Zürich, Switzerland.

E-mail: odonati@gmx.net

COPYRIGHT ๑ 2010 by the Society of Nuclear Medicine, Inc. ter with PET/MRI than with PET/CT regarding lesions larger than $1 \mathrm{~cm}$ in diameter. Compared with Gd-EOB-DTPA-enhanced MRI, PET/MRI resulted in a nonsignificant increase in sensitivity and diagnostic confidence.

Key Words: hepatology; MRI; oncology; PET/MRI; PET/CT; $\mathrm{PET} / \mathrm{MRI}$; liver metastases; multimodality imaging; positron emission tomography

J Nucl Med 2010; 51:692-699

DOI: 10.2967/jnumed.109.068510

$\mathbf{I}_{\mathrm{n}}$ maging of liver metastases is still challenging. In the last few years, dynamic contrast-enhanced CT has become the primary imaging modality for detection of liver metastases, with reported sensitivities of $73 \%-85 \%(1,2) .{ }^{18} \mathrm{~F}-\mathrm{FDG}$ PET has been reported to be superior to CT for detecting liver metastases from colorectal cancer (3) and by some was found the most sensitive noninvasive imaging investigation for detecting liver metastases $(4,5) .{ }^{18} \mathrm{~F}-\mathrm{FDG}$ PET/ $\mathrm{CT}$ has been performed with the objective of improving detectability and increasing diagnostic performance and has shown sensitivities of between $61 \%$ and $97 \%$ in the detection of liver metastases (6-10).

Dynamic contrast-enhanced MRI techniques improve diagnostic accuracy in detecting liver metastases $(11,12)$. Rapid sequential imaging with gadolinium-based contrast agents in the extracellular space is superior to unenhanced imaging and helical CT for detecting metastatic liver disease (13). With the recent availability of hepatobiliary contrast medium with uptake into hepatocytes, the detectability and characterization of liver lesions and especially small liver metastases have further improved (14-18).

For overall patient management, ${ }^{18} \mathrm{~F}-\mathrm{FDG}$ PET and PET/ CT have the added advantage over MRI and CT of providing not only anatomic but also functional information. 
Thus, improved assessment of therapeutic response becomes possible, as ${ }^{18} \mathrm{~F}$-FDG activity is seen only in vital tumor cells (19). CT and MRI rely on only the indirect Response Evaluation Criteria in Solid Tumors to assess therapeutic response. The combination of functional information derived from ${ }^{18} \mathrm{~F}-\mathrm{FDG}$ PET with anatomic information derived from MRI may be of further help in the detection and characterization of liver metastases and in preoperative planning and therapeutic follow-up of patients with liver metastases from gastrointestinal or pancreatic cancer. Compared with using ${ }^{18}$ F-FDG PET alone, combining the advantages of ${ }^{18} \mathrm{~F}$-FDG PET (detection of extrahepatic disease and evaluation of therapeutic response) with the advantages of MRI (higher spatial resolution and tissue contrast) may lead to improved lesion detection and characterization (20).

The purpose of this study was to compare the accuracy and diagnostic confidence of PET/CT, gadolinium-ethoxybenzyldiethylenetriamine pentaacetic acid (Gd-EOB-DTPA)enhanced MRI, and retrospectively fused PET and MRI (PET/MRI) for depiction of liver lesions. Surgery and histologic analysis served as the standard of reference.

\section{MATERIALS AND METHODS}

This study followed Declaration of Helsinki principles (21) and was approved by the ethical review board at our institution. Informed patient consent was waived for this retrospective evaluation.

\section{Patients}

We retrospectively included 51 consecutive patients, in whom PET/CT and Gd-EOB-DTPA-enhanced MRI of the liver had been performed within $30 \mathrm{~d}$ and a clear standard of reference for hepatic lesions was available (Supplemental Fig. 1; supplemental materials are available online only at http://jnm.snmjournals.org).

\section{Data Acquisition}

PET/CT and Gd-EOB-DTPA-enhanced MR images were acquired separately without special prerequisites with regard to the planned image fusion.

\section{$\mathrm{PET} / \mathrm{CT}$}

All data were acquired on 1 of 2 combined PET/CT in-line systems (Discovery STE or Discovery RX; GE Healthcare).

The patients fasted for at least $4 \mathrm{~h}$ before scanning, which started approximately 45-60 min after the injection of 370-400 $\mathrm{MBq}$ of ${ }^{18} \mathrm{~F}-\mathrm{FDG}$. The glucose level (range, $80-120 \mathrm{mg} / \mathrm{dL}$ [4.4$6.7 \mathrm{mmol} / \mathrm{L}]$ ) was measured before scanning. Patients with elevated glucose levels were rescheduled and scanned when glucose levels were normal. Patients were examined in the supine position. Initially, a low-dose CT scan was acquired starting from the level of the head using the following parameters: $40 \mathrm{mAs}, 140 \mathrm{kV}, 0.5 \mathrm{~s} /$ tube rotation, a slice thickness of $4.25 \mathrm{~mm}$, a scan length of $867 \mathrm{~mm}$, and a data acquisition time of $22.5 \mathrm{~s}$. The CT scan was acquired during breath holding in the normal expiratory position. The CT data were used for attenuation correction and lesion localization. PET raw data were iteratively reconstructed using a standard algorithm: ordered-subset expectation maximization (28 subsets, 2 iterative steps) for 2-dimensional (2D) PET, and a fully 3-dimensional (3D) ordered-subset expectation maximization iterative reconstruction algorithm for 3D PET.

Immediately after the $\mathrm{CT}$ acquisition, PET emission scans were acquired with a time of $90 \mathrm{~s}$ to 3 min per cradle position with a 1 -slice overlap in $2 \mathrm{D}$ and a 7 -slice overlap in $3 \mathrm{D}$ reconstruction mode (matrix, $128 \times 128$ ). Acquisition time per cradle position was $3 \mathrm{~min}$ in $2 \mathrm{D}$ mode and $1.5 \mathrm{~min}$ in $3 \mathrm{D}$ mode. The $8-9$ cradle positions starting from the head and continuing to the knees resulted in an acquisition time of approximately 12-27 min.

\section{Gd-EOB-DTPA-Enhanced MRI}

All individuals underwent MRI of the liver using a 1.5-T MRI system (Signa EchoSpeed EXCITE HD; GE Healthcare). For signal reception, an 8-channel anteroposterior phased-array surface coil was placed around the individual and covered the entire liver. Imaging protocols included unenhanced and contrast-enhanced sequences. Unenhanced sequences consisted of single-shot fast spin-echo, T2-weighted fast spin-echo, and in- and out-of-phase gradient-echo sequences. Contrast-enhanced MRI was performed during the hepatic arterial, portal venous, and extracellular phases after intravenous injection of Gd-EOB-DTPA (gadoxetic acid [Primovist; Bayer Schering Pharma]) at a dose of $25 \mu \mathrm{mol} / \mathrm{kg}$ of body weight and a flow rate of $2 \mathrm{~mL} / \mathrm{s}$, followed by a $20-\mathrm{mL}$ saline flush. In addition, delayed imaging in the hepatospecific phase of the contrast agent was performed 20 and 30 min after contrast injection. Contrast-enhanced MRI was performed using a breath-hold fat-suppressed 3D T1-weighted gradient-echo sequence with parallel imaging. Imaging parameters and plane orientations are displayed in Table 1.

\section{Image Registration}

For image registration and fusion, a software prototype, currently under development and not commercially available in any markets yet, was used (Integrated Registration; GE Healthcare). This software is being developed with the goal of becoming a multimodality registration package. Its intended use is for automatic propagation of registration across series acquired in the same examination based on 3 registration methods: automatic, manual, and landmark-based. These can be combined to provide an optimal result. In our study, we used an automatic quaternionbased algorithm for rigid registration.

\section{Image Analysis}

Images were analyzed on a dedicated workstation (Advantage Windows, version 4.3; GE Healthcare Europe). Three sets of images were analyzed for each patient: PET/CT (image set A), unenhanced and Gd-EOB-DTPA-enhanced MRI (image set B), and unenhanced and Gd-EOB-DTPA-enhanced MRI with PET/ MRI (image set C). Separately and independently, 1 experienced nuclear medicine physician and radiologist (reader 1) evaluated image set $\mathrm{A}$, and 1 experienced radiologist (reader 2) evaluated image set B. Subsequently, in a second image reading session, both readers evaluated image set $\mathrm{C}$. When the readers evaluated image set $\mathrm{C}$, they had available image set $\mathrm{B}$, the PET part of the PET/CT dataset, and the fused PET/MRI dataset (Supplemental Fig. 2). To minimize any recall bias, the reading sessions were separated by $5 \mathrm{wk}$. Neither reader was aware of the results of other imaging studies, the results of the other reader, or the findings of surgery or histopathologic examination.

All images of each image set were evaluated in random order on an Advantage Windows workstation. 
TABLE 1. Acquisition Parameters for Gd-EOB-DTPA-Enhanced MRI

\begin{tabular}{|c|c|c|c|c|}
\hline Parameter & T2-SSFSE & T2-FSE & T1-FSPGR & T1-3D-GRE \\
\hline Imaging plane & Coronal & Transverse & Transverse & Transverse \\
\hline Repetition time/echo time (ms) & $1,119 / 88$ & $9,474 / 89$ & $135 / 4.7$ and 2.2 & $3.1 / 1.4$ \\
\hline Inversion recovery time (ms) & NA & NA & NA & 7 \\
\hline Flip angle (degrees) & NA & 90 & 60 & 15 \\
\hline Matrix size & $384 \times 224$ & $256 \times 224$ & $224 \times 192$ & $384 \times 256$ \\
\hline Section thickness $(\mathrm{mm})$ & 5 & 5 & 5 & 4 \\
\hline Intersection gap (mm) & 1 & 1 & 1 & None \\
\hline Overlap (mm) & None & None & None & 2 \\
\hline No. of signals acquired & 0.5 & 2 & 1 & 0.73 \\
\hline Parallel imaging acceleration factor & NA & NA & NA & 2 \\
\hline Receiver bandwidth (kHz) & 62.5 & 50 & 50 & 83.3 \\
\hline
\end{tabular}

In image sets $\mathrm{A}, \mathrm{B}$, and $\mathrm{C}$, the presence, location, and size of any liver lesions were recorded using a grid reference and segmental location. The diameters of the lesions were measured, and confidence of each liver lesion with regard to malignancy was assessed using a 5-point Likert scale (1, definitely benign; 2, probably benign; 3 , indeterminate; 4 , probably malignant; 5 , definitely malignant).

In determining whether a tumor was malignant or benign on Gd-EOB-DTPA-enhanced MRI, the readers used the criteria of Reimer et al. (22). At PET/CT, suggestive lesions were defined as previously reported by Rappeport et al. (23). Standardized uptake values were not calculated.

\section{Standard of Reference}

The imaging findings were carefully correlated to surgical and pathologic records by an author other than those who performed the masked reading. All patients with malignant liver lesions $(n=$ $24)$ underwent hemihepatectomy or segmental resection $(n=19)$, postmortem autopsy $(n=2)$ or open biopsy $(n=3)$. Patients with only benign liver lesions $(n=5)$ underwent imaging follow-up $(n=3$; mean $\pm \mathrm{SD}, 21.2 \pm 6.3 \mathrm{mo})$, postmortem autopsy $(n=1)$, or intraoperative biopsy $(n=1)$ (Supplemental Fig. 1).

\section{Statistical Analysis}

Methods were compared on a per-lesion and per-patient basis. Lesion detectability was determined regardless of evaluation of malignancy of the respective lesion. Sensitivity and specificity in detecting and characterizing liver metastases were determined by counting as liver metastases only those lesions that were graded 4 or 5. To address clustering of lesions within patients, lesion detection rates, sensitivities, and specificities were compared between PET/CT and Gd-EOB-DTPA-enhanced MRI using the multilevel mixed-effects logistic regression routine xtmelogit of Stata (Intercooled Stata, version 10.0; Stata Corp.) for Macintosh (Apple Inc.). A receiver-operating-characteristic curve was fitted to each reader's confidence rating for liver lesions. The accuracy of the 3 methods and the 2 readers was estimated by calculating the area under the receiver-operating-characteristic curve (AUC).

Patient-based lesion detectability, sensitivity, and specificity were analyzed using the McNemar test. For the analysis of confidence for classification of benign and malignant lesions, a confidence level of 1 was considered best confidence in benign lesions, whereas a confidence level of 5 was considered best confidence in malignant lesions. To address clustering of lesions within patients, mean confidence within patients was computed and analyzed using the Wilcoxon signed rank test. A $P$ value of less than 0.05 was considered to indicate a statistically significant difference. The unweighted k-statistic with binary data was used to assess interreader variability in the assessment of PET/MRI. Binary values of 0 (not malignant) and 1 (malignant) were assigned to lesions with a malignancy rating of 3 or less and to lesions with a rating of 4 or more, respectively. Statistical analyses were performed with statistical software SPSS (version 15.0; SPSS Inc.) and Stata.

\section{RESULTS}

\section{Patients}

Of the 51 patients, 14 were excluded because they underwent surgery $(n=3)$ or chemo- or radiotherapy less than $30 \mathrm{~d}$ before imaging $(n=6)$ or between examinations $(n=5)$. The final study group comprised 37 patients $(23$ men and 14 women) with a mean age of 60.2 y (range, 35$82 \mathrm{y}$ ). The mean interval between PET/CT and Gd-EOBDTPA-enhanced MRI was $11.9 \pm 9 \mathrm{~d}$, and the range was 0-30 d. Nineteen $(51 \%)$ of the 37 patients never had chemo- or radiotherapy before imaging. The remaining $18(49 \%)$ of 37 patients had chemo- or radiotherapy between 32 and 808 d before imaging (mean, $209.7 \pm$ $226 \mathrm{~d})$.

The primary tumor that initiated evaluation of the patient for liver metastasis was colorectal cancer $(n=20)$; pancreatic cancer $(n=11)$; ear, nose, and throat cancer $(n=1)$; breast cancer $(n=1)$; lung cancer $(n=1)$; hepatocellular carcinoma $(n=1)$; cholangiocellular carcinoma $(n=1)$; or melanoma $(n=1)$.

\section{Liver Lesions}

A total of 85 liver lesions (55 liver metastases and 30 benign lesions) were present in 29 patients. In 8 patients, no liver lesion was present. Five patients had only benign lesions. The size distribution of lesions is shown in Table 2. Benign lesions included liver cysts $(n=18)$, liver cell adenomas $(n=5)$, focal nodular hyperplasias $(n=4)$, and 
TABLE 2. Size Distribution of the 85 Hepatic Lesions

\begin{tabular}{ccc}
$\begin{array}{c}\text { Diameter } \\
(\mathrm{mm})\end{array}$ & $\begin{array}{c}\text { Number } \\
\text { of metastases }\end{array}$ & $\begin{array}{c}\text { Number of } \\
\text { lesions total }\end{array}$ \\
$\leq 5$ & $1(2 \%)$ & $6(7 \%)$ \\
$6-10$ & $9(16 \%)$ & $18(21 \%)$ \\
$11-20$ & $22(40 \%)$ & $35(41 \%)$ \\
$\geq 21$ & $23(42 \%)$ & $26(31 \%)$ \\
Total & $55(100 \%)$ & $85(100 \%)$ \\
\hline
\end{tabular}

postoperative lesions $(n=3)$. Twenty-four patients had a total of 55 liver metastases (mean, $2.3 \pm 2.1$; range, 1-8). All 24 of those patients underwent definitive surgery, operative biopsy, or postmortem autopsy with histopathologic examination. Forty-five liver metastases were confirmed histologically in these 24 patients. The remaining 10 liver metastases were confirmed by follow-up imaging (mean, $11.9 \pm 2.7 \mathrm{mo}$ ).

\section{Lesion Detectability}

Regarding all 85 lesions (independent of the diameter of the lesion), detection rate in determining the presence of a lesion differed significantly between PET/CT and Gd-EOBDTPA-enhanced MRI (64\% and $85 \% ; P=0.002)$.

For lesions $1 \mathrm{~cm}$ in diameter or smaller, the difference in detection rate between PET/CT and Gd-EOB-DTPAenhanced MRI was significant $(29 \%$ and $71 \% ; P=0.013)$ (Fig. 1).

Also for lesions larger than $1 \mathrm{~cm}$ in diameter, the difference in detection rate between PET/CT and GdEOB-DTPA-enhanced MRI was significant $(77 \%$ and $90 \% ; P=0.03$ ) (Table 3).

\section{Sensitivity and Specificity in Detection of Liver Metastases}

Regarding all 85 lesions (benign and malignant combined), consistent with the higher detectability, the sensitivity in detecting and correctly characterizing liver metastases was significantly higher for PET/MRI than for PET/CT (93\% and 76\%; $P=0.023$ ). The sensitivity of GdEOB-DTPA-enhanced MRI (91\%) was not significantly different from that of PET/MRI (Table 4).

For the subgroups of lesions $1 \mathrm{~cm}$ in diameter or smaller $(n=24)$, the sensitivities for PET/CT, Gd-EOB-DTPAenhanced MRI, and PET/MRI were 30\% (3/10), 80\% (8/ 10 ), and $70 \%$ (7/10), respectively. Specificities were $86 \%$ $(12 / 14)$ and $100 \%(14 / 14)$ for PET/CT and Gd-EOBDTPA-enhanced MRI. For PET/MRI, specificities were $71 \%(10 / 14)$ for reader 1 and $100 \%$ (14/14) for reader 2. There were too few lesions to reveal significant differences between methods (Table 4).

For lesions larger than $1 \mathrm{~cm}$, the sensitivities for PET/CT, Gd-EOB-DTPA-enhanced MRI, and PET/MRI were 87\% (39/45), 93\% (42/45), and 98\% (44/45), respectively. Specificities were $94 \%(15 / 16)$ and $100 \%(16 / 16)$ for PET/CT and Gd-EOB-DTPA-enhanced MRI. For PET/ MRI, specificities were $100 \%(16 / 16)$ for reader 1 and

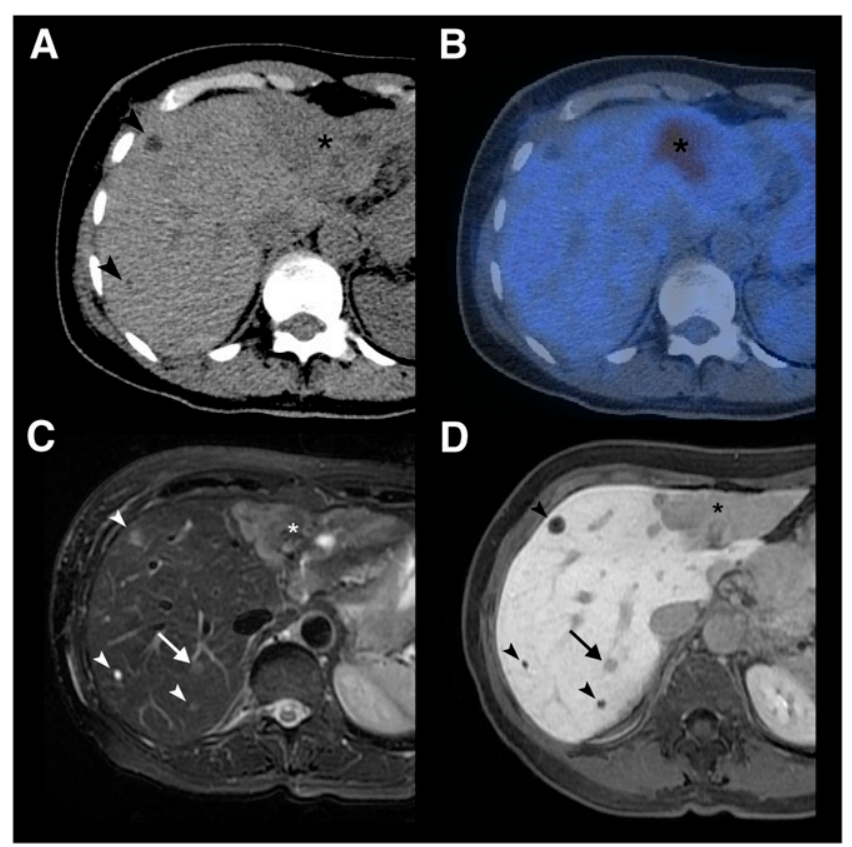

FIGURE 1. A 64-y-old patient with multiple liver metastases due to breast cancer. (A) On CT, 2 small (arrowheads) and 1 large (asterisk) hypodense lesion are noted. (B) Only the large lesion, in liver segment II/III, shows ${ }^{18} \mathrm{~F}-\mathrm{FDG}$ uptake on PET/CT. (C and D) In liver segment VII, 2 additional lesions not visible on CT and PET/CT images are seen on T2-weighted fast spin-echo MRI (C) and Gd-EOB-DTPAenhanced 3D gradient-echo MRI in hepatobiliary phase (D). One lesion (arrow) corresponds to metastasis as confirmed by intraoperative biopsy; other lesion (arrowhead) corresponds to cysts.

94\% (15/16) for reader 2 (Table 4). Differences between modalities were not significant.

On a per-patient basis, the sensitivity of PET/CT, GdEOB-DTPA-enhanced MRI, PET/MRI for reader 1, and PET/MRI for reader 2 was $100 \%, 96 \%, 96 \%$, and $100 \%$, respectively. The respective specificity values were $92 \%$, $100 \%, 92 \%$, and $100 \%$.

TABLE 3. Detection Rate for Liver Lesions (Benign and Malignant)

Lesion group and modality Detection rate (\%)

All lesions

$\mathrm{PET} / \mathrm{CT}$

Gd-EOB-DTPA-enhanced MRI

Lesions $\leq 1 \mathrm{~cm}$ in diameter

PET/CT

Gd-EOB-DTPA-enhanced MRI

Lesions $>1 \mathrm{~cm}$ in diameter

$\mathrm{PET} / \mathrm{CT}$

Gd-EOB-DTPA-enhanced MRI

$64^{*}$

85

$29^{*}$

71

$77^{\star}$

90

${ }^{*}$ Considering all subgroups, differences in lesion detection were significant $(P<0.05)$ between PET/CT and Gd-EOBDTPA-enhanced MRI. 


\begin{tabular}{|c|c|c|}
\hline Lesion group and modality & $\begin{array}{c}\text { Sensitivity } \\
(\%)\end{array}$ & $\begin{array}{c}\text { Specificity } \\
(\%)\end{array}$ \\
\hline \multicolumn{3}{|l|}{ All lesions } \\
\hline PET/CT & $76^{\star}$ & 90 \\
\hline Gd-EOB-DTPA-enhanced MRI & 91 & 100 \\
\hline PET/MRI ${ }^{\dagger}$ & $93 / 93$ & $87 / 97$ \\
\hline \multicolumn{3}{|l|}{ Lesions $\leq 1 \mathrm{~cm}$ in diameter } \\
\hline PET/CT & 30 & 86 \\
\hline Gd-EOB-DTPA-enhanced MRI & 80 & 100 \\
\hline $\mathrm{PET} / \mathrm{MRI}{ }^{\dagger}$ & $70 / 70$ & $71 / 100$ \\
\hline \multicolumn{3}{|l|}{ Lesions $>1 \mathrm{~cm}$ in diameter } \\
\hline PET/CT & 87 & 94 \\
\hline Gd-EOB-DTPA-enhanced MRI & 93 & 100 \\
\hline PET/MRI ${ }^{\dagger}$ & $98 / 98$ & $100 / 94$ \\
\hline \multicolumn{3}{|c|}{$\begin{array}{l}{ }^{*} \text { Difference in sensitivity was significant }(P<0.05) \text { between } \\
\text { PET/CT and PET/MRI when all liver metastases were consid- } \\
\text { ered. } \\
{ }^{\dagger} \text { Values are for reader } 1 / \text { reader } 2 \text {, respectively. }\end{array}$} \\
\hline
\end{tabular}

\section{Accuracy in Lesion Characterization}

When all 85 lesions were considered, the AUC was higher for Gd-EOB-DTPA-enhanced MRI than for PET/CT (0.94 and 0.85, respectively). For reader 1, AUC was higher for PET/MRI than for PET/CT (0.92 and 0.85, respectively). For reader 2, AUC was 0.94 and 0.96 for Gd-EOB-DTPAenhanced MRI and PET/MRI, respectively. Regardless of lesion size, PET/MRI was the most accurate technique for both readers (Supplemental Fig. 3).

For lesions $1 \mathrm{~cm}$ in diameter or smaller, Gd-EOBDTPA-enhanced MRI showed a higher AUC than did PET/CT (0.84 and 0.54). For reader 1, AUC was higher for PET/MRI than for PET/CT (0.66 and 0.54, respectively). For reader 2, AUC increased only slightly when PET information was added to MRI (Gd-EOB-DTPAenhanced MRI and PET/MRI, 0.84 and 0.87, respectively).

For reader 1, the AUC for lesions larger than $1 \mathrm{~cm}$ was higher for PET/MRI than for PET/CT (0.99 and 0.93, respectively). The increase in the AUC for reader 2 between Gd-EOB-DTPA-enhanced MRI and PET/MRI was slight (0.96 and 0.97, respectively) (Table 5).

\section{Diagnostic Confidence}

Evaluation of diagnostic confidence regarding all benign and malignant lesions larger than $1 \mathrm{~cm}$ showed a significant difference between PET/CT and PET/MRI. On a per-lesion basis, reader 1 was more confident with PET/CT in 7 cases and more confident with PET/MRI in 16 cases (Supplemental Fig. 4). In 38 cases, the level of confidence remained the same ( $P=0.046$ in patient-based analysis).

Considering all lesions, the diagnostic confidence between PET/CT and Gd-EOB-DTPA-enhanced MRI and between PET/MRI and Gd-EOB-DTPA-enhanced MRI did not reveal significant differences, but in selected cases,

\section{TABLE 5. AUC in Detection of Liver Metastases}

Lesion group and modality AUC

All lesions

PET/CT

Gd-EOB-DTPA-enhanced MRI $\mathrm{PET} / \mathrm{MRI}{ }^{*}$

0.85

0.94

$0.92 / 0.96$

Lesions $\leq 1 \mathrm{~cm}$ in diameter

$\mathrm{PET} / \mathrm{CT}$

0.54

Gd-EOB-DTPA-enhanced MRI

0.84

PET/MRI*

$0.66 / 0.87$

Lesions $>1 \mathrm{~cm}$ in diameter

$\mathrm{PET} / \mathrm{CT}$

0.93

Gd-EOB-DTPA-enhanced MRI

0.96

$\mathrm{PET} / \mathrm{MRI}{ }^{*}$

$0.99 / 0.97$

*Values are for reader $1 /$ reader 2 , respectively.

PET/MRI could improve diagnostic confidence over GdEOB-DTPA-enhanced MRI (Figs. 2 and 3).

\section{Interreader Variability}

Reader agreement was assessed for the 85 lesions. There was good agreement in grading liver lesions as benign or malignant on PET/MRI, with a $\kappa$-value of 0.72 .

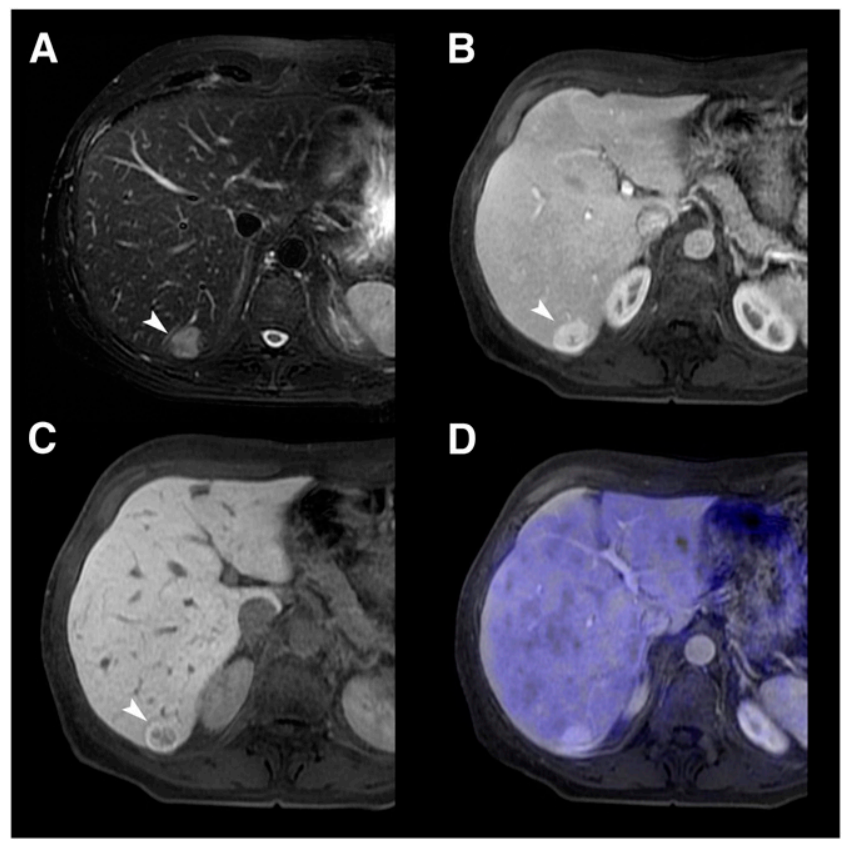

FIGURE 2. A 56-y-old patient with incidental liver finding on sonography. (A) T2-weighted MRI shows hyperintense lesion (arrowhead) in segment VI. (B and C) On Gd-EOBDTPA-enhanced MRI, lesion shows contrast uptake during portal venous phase (B) and no contrast uptake during hepatobiliary phase (C). Lesion was first graded as indeterminate (grade 3). When information from PET was added to $\mathrm{MRI}(\mathrm{D})$, lack of ${ }^{18} \mathrm{~F}-\mathrm{FDG}$ uptake indicated that lesion was definitely benign (grade 1). Histopathology confirmed diagnosis of focal nodular hyperplasia. 


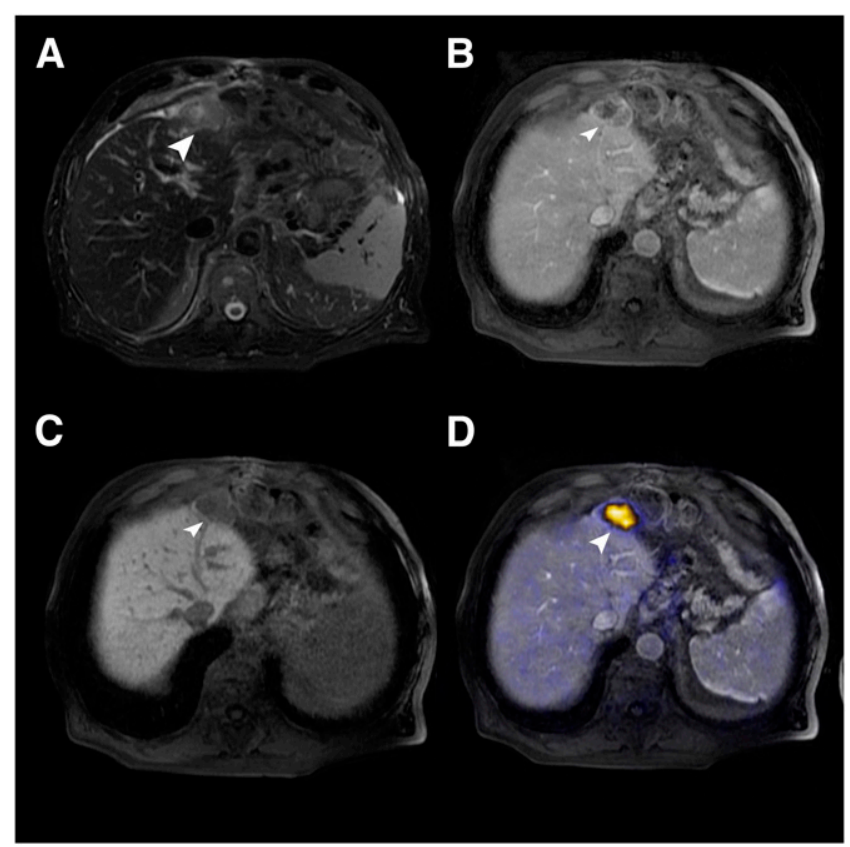

FIGURE 3. An 82-y-old gastric cancer patient after left hemihepatectomy. (A) On T2-weighted fast spin-echo MRI, subcapsular lesion (arrowhead) is hyperintense. (B) Lesion shows slight rimlike contrast uptake during portal venous phase. (C) Lesion shows no contrast medium uptake during hepatobiliary phase after injection of Gd-EOB-DTPA. Based on MRI findings, lesion was graded as probably malignant (grade 4). (D) When information from PET was added to MRI, ${ }^{18} \mathrm{~F}-\mathrm{FDG}$ uptake indicated that lesion was definitely malignant (grade 5). Histopathology confirmed diagnosis of hepatic metastasis from gastric carcinoma.

\section{DISCUSSION}

Our results demonstrate a superior detection rate and higher confidence in characterization of liver metastases for Gd-EOB-DTPA-enhanced MRI or PET/MRI than for PET/ CT. Gd-EOB-DTPA-enhanced MRI was significantly more sensitive in the detection of liver lesions than was PET/CT. Also, sensitivity in detection and correct classification of lesions as malignant was significantly better for either of the 2 MRI-based techniques than for PET/CT. However, a significant difference in sensitivity between Gd-EOBDTPA-enhanced MRI and PET/MRI could not be observed. Several factors contribute to the higher sensitivity of the MRI-based modalities than of PET/CT. The most important factor is the higher spatial resolution of MRI than of ${ }^{18} \mathrm{~F}-\mathrm{FDG}$ PET and the better soft-tissue contrast in comparison to PET/CT. Second, the use of hepatocyte-specific contrast medium additionally improves the sensitivity of Gd-EOB-DTPA-enhanced MRI and PET/MRI.

The $76 \%$ sensitivity of PET/CT in detecting liver metastases in our study lies within the wide range of reported sensitivities and might have been improved further if PET/ CT had been performed with dedicated contrast-enhanced CT. Recent studies (3,24-26) and metaanalyses $(4,5)$ of
PET for detecting liver metastases have reported sensitivities from $61 \%$ to $94 \%$. The few studies that have investigated the sensitivity of PET/CT in detection of liver metastases have reported values between $61 \%$ and $97 \%$ (610,27). The fact that our sensitivity for PET/CT is rather at the lower end of the range may be explained in part by the relatively high number of liver metastases with a diameter of $1 \mathrm{~cm}$ or less (18\%). From the studies mentioned above, $\mathrm{PET} / \mathrm{CT}$ is known to have a relatively low sensitivity in the detection of liver metastases smaller than $1 \mathrm{~cm}$. Our reported PET/CT sensitivity of $30 \%$ for small lesions is comparable to the sensitivity found by Coenegrachts et al., who reported a detection rate of $36 \%$ for liver metastases smaller than $1 \mathrm{~cm} \mathrm{(10).}$

Only a few studies have compared lesion detection and characterization between contrast-enhanced MRI and PET/ CT $(20,23,28,29)$, but in none of them was Gd-EOB-DTPA used as the liver-specific contrast medium. Our results for the sensitivity of Gd-EOB-DTPA-enhanced MRI are comparable to results from studies examining detection of metastasis using MRI with liver-specific contrast agents, superparamagnetic iron oxide in most (18,23,29-32). Sahani et al. retrospectively compared mangafodipir trisodiumenhanced MRI of the liver with whole-body ${ }^{18} \mathrm{~F}-\mathrm{FDG}$ PET in patients with adenocarcinoma of the colon or pancreas (29). Their reported sensitivities of $67 \%$ for PET and $81 \%$ for MRI were slightly lower than the sensitivities in our presented study. The marked increase in sensitivity of Gd-EOB-DTPA-enhanced MRI and PET/MRI over $\mathrm{PET} / \mathrm{CT}$ in lesions measuring $1 \mathrm{~cm}$ or less in diameter is certainly due to the higher soft-tissue contrast of MRI than of PET/CT, as several studies have demonstrated $(14,28,33)$. In addition, especially small liver lesions are hard to detect if no intravenous contrast medium is used for PET/CT. Another factor that may conceal these small lesions is the background noise and beam-hardening artifacts that are apparent using low-dose CT for PET/ CT. Therefore, our results for the detection of small hepatic lesions by PET/CT may have been negatively influenced by the applied PET/CT protocol.

PET/MRI has been suggested mainly for brain malignancies so far $(34,35)$, whereas our results show the potential use of PET/MRI in an oncologic setting. To the best of our knowledge, no studies have evaluated detection of liver metastases by retrospective PET/MR image fusion. The already high diagnostic confidence and sensitivity of Gd-EOB-DTPA-enhanced MRI in the detection and characterization of liver metastases showed that neither AUC for diagnostic accuracy nor sensitivity or diagnostic confidence differed significantly between Gd-EOB-DTPAenhanced MRI and PET/MRI. This finding is due to the fact that Gd-EOB-DTPA-enhanced MRI alone already demonstrates high sensitivity and diagnostic accuracy in liver metastasis detection. However, in some cases, PET/ MRI increased diagnostic confidence when compared with Gd-EOB-DTPA-enhanced MRI. 
In liver metastases smaller than $1 \mathrm{~cm}$, in which sensitivity for Gd-EOB-DTPA-enhanced MRI also drops, the addition of PET to Gd-EOB-DTPA-enhanced MRI is not of much benefit as such small lesions rarely show pathologic ${ }^{18}$ F-FDG uptake $(27,36,37)$. However, a significant increase in diagnostic confidence of PET/MRI over PET/ CT can be noted in lesions larger than $1 \mathrm{~cm}$.

One limitation of our study is that PET/CT was obtained using a low-dose CT scan (40 mAs) without the administration of intravenous contrast material. Recently, 1 study has shown an increase in tumor-to-background ratio and hypermetabolic lesion diameter using a dual-time-point acquisition protocol for the liver (8). Therefore, our protocol was not optimized for the detection and characterization of liver metastasis. A multiphase contrast-enhanced CT scan with arterial and portal venous phase and dual-timepoint PET acquisition would be preferred. Further studies, comparing optimized PET/CT versus Gd-EOB-DTPAenhanced MRI and PET/MRI, are needed.

A second limitation of our study is that extrahepatic metastases were not evaluated and therefore no statement can be made about staging of patients. The ability to image the whole body is a major advantage of PET and PET/CT regarding staging and evaluation of extrahepatic disease $(28,38-40)$. In our retrospective study design, we evaluated Gd-EOB-DTPA-enhanced MRI focusing on detection of intrahepatic metastases. Possible extrahepatic disease was not in the field of view of the Gd-EOB-DTPA-enhanced MRI examinations. As of now, a whole-body examination as obtained by PET/CT remains necessary for the detection of extrahepatic disease. Once hybrid PET/MRI systems for body imaging are commercially available, they will enable high sensitivity and specificity in the detection of liver metastases and will detect extrahepatic disease in the same examination-a promising feature for the evaluation of potential surgical candidates.

A third limitation of our study is its retrospective nature. However, the fact that our study was retrospective allowed us to show the feasibility of and good visual results for retrospective image fusion, even in patients for whom fusion of PET and Gd-EOB-DTPA-enhanced MRI was not intended at the time of acquisition and for whom, therefore, no specially prepared imaging protocols were applied.

A fourth limitation is the relatively small size of our patient sample. Our sample was small mainly for 2 reasons. First, Gd-EOB-DTPA is a relatively new contrast agent and therefore has been used in only a limited number of examinations. Second, we set a relatively short interval of $30 \mathrm{~d}$ between the PET/CT and Gd-EOB-DTPA-enhanced MRI acquisitions. This short interval was necessary to minimize the growth of liver metastases between imaging studies.

A final limitation is the inclusion of a patient who had hepatocellular carcinoma as the primary tumor. Hepatocellular carcinoma lesions are less ${ }^{18} \mathrm{~F}-\mathrm{FDG}$-avid than other malignancies. However, this particular patient did not present any secondary liver lesions and therefore did not falsify the results.

\section{CONCLUSION}

Our results suggest that, compared with PET/CT, GdEOB-DTPA-enhanced MRI and PET/MRI are more sensitive in the detection of liver metastases. The increase in detection is especially relevant in small liver metastases $1 \mathrm{~cm}$ or less in diameter. For lesions larger than $1 \mathrm{~cm}$ in diameter, diagnostic confidence was significantly better with PET/MRI than with PET/CT. However, compared with Gd-EOB-DTPA-enhanced MRI, the addition of functional information from PET did not significantly increase sensitivity or diagnostic confidence.

\section{REFERENCES}

1. Furuhata T, Okita K, Tsuruma T, et al. Efficacy of SPIO-MR imaging in the diagnosis of liver metastases from colorectal carcinomas. Dig Surg. 2003;20: 321-325.

2. Valls C, Andia E, Sanchez A, et al. Hepatic metastases from colorectal cancer: preoperative detection and assessment of resectability with helical CT. Radiology. 2001;218:55-60.

3. Delbeke D, Vitola JV, Sandler MP, et al. Staging recurrent metastatic colorectal carcinoma with PET. J Nucl Med. 1997;38:1196-1201.

4. Bipat S, van Leeuwen MS, Comans EF, et al. Colorectal liver metastases: CT, MR imaging, and PET for diagnosis-meta-analysis. Radiology. 2005;237: 123-131.

5. Kinkel K, Lu Y, Both M, Warren RS, Thoeni RF. Detection of hepatic metastases from cancers of the gastrointestinal tract by using noninvasive imaging methods (US, CT, MR imaging, PET): a meta-analysis. Radiology. 2002;224:748-756.

6. Votrubova J, Belohlavek O, Jaruskova M, et al. The role of FDG-PET/CT in the detection of recurrent colorectal cancer. Eur J Nucl Med Mol Imaging. 2006;33: 779-784.

7. Nakamoto Y, Sakamoto S, Okada T, et al. Clinical value of manual fusion of PET and CT images in patients with suspected recurrent colorectal cancer. AJR. 2007; 188:257-267.

8. Dirisamer A, Halpern BS, Schima W, et al. Dual-time-point FDG-PET/CT for the detection of hepatic metastases. Mol Imaging Biol. 2008;10:335-340.

9. Chua SC, Groves AM, Kayani I, et al. The impact of ${ }^{18} \mathrm{~F}-\mathrm{FDG}$ PET/CT in patients with liver metastases. Eur J Nucl Med Mol Imaging. 2007;34: 1906-1914.

10. Coenegrachts K, De Geeter F, ter Beek L, et al. Comparison of MRI (including SS SE-EPI and SPIO-enhanced MRI) and FDG-PET/CT for the detection of colorectal liver metastases. Eur Radiol. 2009;19:370-379.

11. Hawighorst H, Schoenberg SO, Knopp MV, Essig M, Miltner P, van Kaick G. Hepatic lesions: morphologic and functional characterization with multiphase breath-hold 3D gadolinium-enhanced MR angiography-initial results. Radiology. 1999;210:89-96.

12. Semelka RC, Cance WG, Marcos HB, Mauro MA. Liver metastases: comparison of current MR techniques and spiral CT during arterial portography for detection in 20 surgically staged cases. Radiology. 1999;213:86-91.

13. Ward J. New MR techniques for the detection of liver metastases. Cancer Imaging. 2006;6:33-42.

14. Bluemke DA, Sahani D, Amendola M, et al. Efficacy and safety of MR imaging with liver-specific contrast agent: U.S. multicenter phase III study. Radiology. 2005;237:89-98.

15. Huppertz A, Balzer T, Blakeborough A, et al. Improved detection of focal liver lesions at MR imaging: multicenter comparison of gadoxetic acid-enhanced MR images with intraoperative findings. Radiology. 2004;230:266-275.

16. Kim YK, Ko SW, Hwang SB, Kim CS, Yu HC. Detection and characterization of liver metastases: 16-slice multidetector computed tomography versus superparamagnetic iron oxide-enhanced magnetic resonance imaging. Eur Radiol. 2006;16:1337-1345.

17. Lee HY, Lee JM, Kim SH, et al. Detection and characterization of focal hepatic lesions: comparative study of MDCT and gadobenate dimeglumine-enhanced MR imaging. Clin Imaging. 2008;32:287-295.

18. Reimer P, Jahnke N, Fiebich $\mathrm{M}$, et al. Hepatic lesion detection and characterization: value of nonenhanced MR imaging, superparamagnetic iron 
oxide-enhanced MR imaging, and spiral CT-ROC analysis. Radiology. 2000;217: $152-158$.

19. Cascini GL, Avallone A, Delrio P, et al. ${ }^{18}$ F-FDG PET is an early predictor of pathologic tumor response to preoperative radiochemotherapy in locally advanced rectal cancer. J Nucl Med. 2006;47:1241-1248.

20. Yang M, Martin DR, Karabulut N, Frick MP. Comparison of MR and PET imaging for the evaluation of liver metastases. J Magn Reson Imaging. 2003;17: 343-349.

21. World Medical Association Declaration of Helsinki: ethical principles for medical research involving human subjects. JAMA. 2000;284:3043-3045.

22. Reimer P, Rummeny EJ, Daldrup HE, et al. Enhancement characteristics of liver metastases, hepatocellular carcinomas, and hemangiomas with Gd-EOB-DTPA: preliminary results with dynamic MR imaging. Eur Radiol. 1997;7:275-280.

23. Rappeport ED, Loft A, Berthelsen AK, et al. Contrast-enhanced FDG-PET/CT vs. SPIO-enhanced MRI vs. FDG-PET vs. CT in patients with liver metastases from colorectal cancer: a prospective study with intraoperative confirmation. Acta Radiol. 2007;48:369-378.

24. Rohren EM, Paulson EK, Hagge R, et al. The role of F-18 FDG positron emission tomography in preoperative assessment of the liver in patients being considered for curative resection of hepatic metastases from colorectal cancer. Clin Nucl Med. 2002;27:550-555.

25. Bohm B, Voth M, Geoghegan J, et al. Impact of positron emission tomography on strategy in liver resection for primary and secondary liver tumors. $J$ Cancer Res Clin Oncol. 2004;130:266-272.

26. Truant S, Huglo D, Hebbar M, Ernst O, Steinling M, Pruvot FR. Prospective evaluation of the impact of $\left[{ }^{18} \mathrm{~F}\right]$ fluoro-2-deoxy-D-glucose positron emission tomography of resectable colorectal liver metastases. Br J Surg. 2005;92:362-369.

27. Selzner M, Hany TF, Wildbrett P, McCormack L, Kadry Z, Clavien PA. Does the novel PET/CT imaging modality impact on the treatment of patients with metastatic colorectal cancer of the liver? Ann Surg. 2004;240:1027-1034.

28. Kong G, Jackson C, Koh DM, et al. The use of ${ }^{18}$ F-FDG PET/CT in colorectal liver metastases: comparison with CT and liver MRI. Eur J Nucl Med Mol Imaging. 2008;35:1323-1329.
29. Sahani DV, Kalva SP, Fischman AJ, et al. Detection of liver metastases from adenocarcinoma of the colon and pancreas: comparison of mangafodipir trisodium-enhanced liver MRI and whole-body FDG PET. AJR. 2005;185: 239-246.

30. Choi JY, Kim MJ, Kim JH, et al. Detection of hepatic metastasis: manganeseand ferucarbotran-enhanced MR imaging. Eur J Radiol. 2006;60:84-90.

31. Rappeport ED, Loft A. Liver metastases from colorectal cancer: imaging with superparamagnetic iron oxide (SPIO)-enhanced MR imaging, computed tomography and positron emission tomography. Abdom Imaging. 2007;32:624-634.

32. Reimer P, Schneider G, Schima W. Hepatobiliary contrast agents for contrastenhanced MRI of the liver: properties, clinical development and applications. Eur Radiol. 2004;14:559-578.

33. Ward J, Robinson PJ, Guthrie JA, et al. Liver metastases in candidates for hepatic resection: comparison of helical CT and gadolinium- and SPIO-enhanced MR imaging. Radiology. 2005;237:170-180.

34. Ledezma CJ, Chen W, Sai V, et al. ${ }^{18}$ F-FDOPA PET/MRI fusion in patients with primary/recurrent gliomas: initial experience. Eur J Radiol. 2009;71:242-248.

35. Schlemmer HP, Pichler BJ, Schmand M, et al. Simultaneous MR/PET imaging of the human brain: feasibility study. Radiology. 2008;248:1028-1035.

36. Ruers TJ, Langenhoff BS, Neeleman N, et al. Value of positron emission tomography with [F-18]fluorodeoxyglucose in patients with colorectal liver metastases: a prospective study. J Clin Oncol. 2002;20:388-395.

37. Fong Y, Saldinger PF, Akhurst T, et al. Utility of ${ }^{18}$ F-FDG positron emission tomography scanning on selection of patients for resection of hepatic colorectal metastases. Am J Surg. 1999;178:282-287.

38. Ong KO, Leen E. Radiological staging of colorectal liver metastases. Surg Oncol. 2007;16:7-14.

39. Arulampalam TH, Francis DL, Visvikis D, Taylor I, Ell PJ. FDG-PET for the pre-operative evaluation of colorectal liver metastases. Eur J Surg Oncol. 2004; 30:286-291.

40. Zealley IA, Skehan SJ, Rawlinson J, Coates G, Nahmias C, Somers S. Selection of patients for resection of hepatic metastases: improved detection of extrahepatic disease with FDG pet. Radiographics. 2001;21(spec no):S55-S69. 